\title{
Nuclear structure with radioactive muonic atoms
}

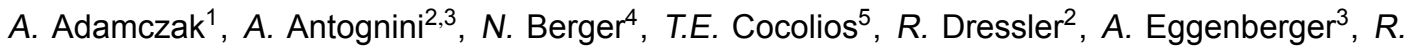

 \\ E. Rapisarda ${ }^{2, \star}$, P. Reiter ${ }^{10}$, N. Ritjoho ${ }^{2,3}$, S. Roccia ${ }^{11}$, N. Severijns ${ }^{5}$, A. Skawran ${ }^{2,3}$, F. Wauters ${ }^{4}$, \\ and $L$. Willmann ${ }^{7}$ \\ ${ }^{1}$ Institute of Nuclear Physics, Polish academy of Sciences, Krakow, Poland \\ ${ }^{2}$ Paul Scherrer Institut, Villigen, Switzerland \\ ${ }^{3}$ ETH Zürich, Switzerland \\ ${ }^{4}$ University of Mainz, Germany \\ ${ }^{5}$ KULeuven, Belgium \\ ${ }^{6}$ LKB Paris, France \\ ${ }^{7} \mathrm{VSI}$, University of Groningen, The Netherlands \\ ${ }^{8}$ University of Victoria, Canada \\ ${ }^{9}$ Perimeter Institute, Waterloo, Canada \\ ${ }^{10}$ Institut für Kernphysik, Universität Köln, Germany \\ ${ }^{11}$ CSNSM, Universitè Paris Sud, CNRS/IN2P3, Orsay Campus, France
}

\begin{abstract}
Muonic atoms have been used to extract the most accurate nuclear charge radii based on the detection of X-rays from the muonic cascades. Most stable and a few unstable isotopes have been investigated with muonic atom spectroscopy techniques. A new research project recently started at the Paul Scherrer Institut aims to extend the highresolution muonic atom spectroscopy for the precise determination of nuclear charge radii and other nuclear structure properties of radioactive isotopes. The challenge to combine the high-energy muon beam with small quantity of stopping mass is being addressed by developing the concept of stopping the muon in a high-density, a high-pressure hydrogen cell and subsequent transfer of the muon to the element of interest. Status and perspectives of the project will be presented.
\end{abstract}

\section{Introduction}

Muonic atoms spectroscopy, i.e. the detection of the muonic X-rays emitted subsequently to the atomic capture of a negative muon, has been a very extensively used technique to determine the extent of the nuclear charge distribution [1]. This method for determining nuclear charge radii complements the knowledge from electron scattering experiments and laser spectroscopy [2].

In muonic atom spectroscopy a negative muon beam is stopped in a target made of the desired material. After slowing down, the muon is captured by the nuclear Coulomb potential, in the so called atomic muon capture, and cascades down from a high initial state around $\mathrm{n} \approx 14$ to the ground state. By measuring the emitted X-ray photons (which for high-Z muonic atoms can reach up to $10 \mathrm{MeV}$ )

\footnotetext{
${ }^{\star}$ e-mail: elisa.rapisarda@psi.ch
} 
the binding energies of the various levels of the muonic atom can be mapped out. Comparing the measured energy levels with calculations taking the finite size of the nucleus into account allows to extract its charge radius. While in principle a single transition energy is sufficient to extract the charge radius, in practice a multitude of transitions is analyzed to constrain the calculations and to determine additional nuclear effects such as nuclear polarization from the data itself. An impressive precision on the charge radius can be achieved amounting in the case of ${ }^{208} \mathrm{~Pb}$ to $0.02 \%$ [3].

After the muon has reached the $1 \mathrm{~s}$ atomic state, it can decay or get captured by the nucleus. The capture of the muon by the nucleus via the weak process

$$
\mu+{ }^{Z} A \rightarrow{ }^{Z-1} A+v_{\mu}
$$

produces "one-step more neutron-rich nuclei" at high excitation energies offering the possibility to gain structural information on neutron-rich nuclei which can be compared with other standard nuclear reactions for obtaining similar or complementary information [4].

The charge radii of basically all stable - and in few exceptional cases unstable - isotopes have been determined [2]. The measurements with muons were limited to isotopes available in gram quantities due to the fact that a muon beam with a momentum of several tens of $\mathrm{MeV} / \mathrm{c}$ needed to be stopped.

Experimentally, it is very challenging to merge the measurements using short-lived muons with isotopes that are available only in small quantities. A promising concept makes use of the transfer mechanism by which negative muons stopped in hydrogen are transferred from the muonic hydrogen to the heavier atoms. It was proposed around 50 years ago to measure transfer rates and root mean square radii with high-pressure gas targets [5]. More recently, to confine the production region of the $\mu \mathrm{Z}$ atoms, Strasser and collaborators $[6,7]$ developed a technique which consists in stopping of muons in a solid hydrogen/deuterium film where the isotope of choice has been embedded. The technique aimed to expand muonic atom spectroscopy to the investigation of the nuclear properties of radioactive nuclei and was designed to be applied in future facilities where both intense negative muon beams and radioactive nuclear beams could be available.

An experimental programme to perform muonic atom spectroscopy of radioactive ions is in progress at the Paul Scherrer Institut (PSI) aiming, as a first test case, at the precise measurement of the nuclear charge radius of ${ }^{226} \mathrm{Ra}$. The precise knowledge of the absolute nuclear charge radius at a level of at least $0.2 \%$ relative precision is needed by the planned measurement of atomic parity violation in a single $\mathrm{Ra}^{+}$ion at the VSI in the Netherlands [8-13]. Because no stable isotopes of Ra exist in nature, only relative differences in mean-square radii along the isotopic chain are available from the optical spectroscopy [14], so far the only method capable of measuring unstable isotopes when only tiny amounts of material are available. The quantity of Ra that can practically be used in the experiment is limited by radiation safety regulations and amounts to $5 \mu \mathrm{g}$, corresponding to a target of $1 \mathrm{~cm}^{2}$ and $10 \mathrm{~nm}$ thickness.

This paper concerns the high resolution gamma-ray spectroscopy of the muonic cascade of unstable atoms and the description of the method being developed at the Paul Scherrer Institut allowing measurements on isotopes that are available in microgram quantities. A brief overview of the concept of muonic atom formation by transfer reaction will be given in Sec.2. A summary of the experimental results is reported in Sec.3 and future plans are outlined in Sec.4.

\section{Muonic atom formation}

The direct atomic muon capture requires the muon to be stopped in the material of interest usually available in quantities of several grams. The project at PSI aims to perform muonic atom spectroscopy on elements available only in microgram quantities and it is inspired by the previous work done in this 
direction (see Introduction). The method being developed uses a double transfer reaction $\mu \mathrm{H} \rightarrow \mu \mathrm{D}$ $\rightarrow \mu \mathrm{Z}$ and the Ramsauer-Townsend effect in a cell filled with hydrogen (H2) and deuterium (D2). The concept is as follows: The muons enter the gas cell and stop forming muonic hydrogen. The admixture of a few percent of deuterium leads to a fast transfer of the muon from the hydrogen to the deuterium nucleus thereby gaining $45 \mathrm{eV}$ of energy. As the muonic deuterium loses energy through repeated collisions with hydrogen the corresponding cross-section drops by close to two orders of magnitude having a minimum at around $4 \mathrm{eV}$ due to the Ramsauer-Townsend effect [15-18]. At this point the gas becomes basically transparent and the muonic deuterium atoms are able to traverse a large distance. If a high- $Z$ target is located at the wall of the cell, the muons are transferred again from the deuterium to the high- $Z$ nuclei. As the corresponding transfer rates are of the order of $10^{12} \mathrm{~s}^{-1}$ only small layers of target material are needed $[19,20]$. In the case of radium a $10 \mathrm{~nm}$ thick layer leads to a transfer probability of around $40 \%$, assuming that the muonic deuterium moves at constant kinetic energy of $4 \mathrm{eV}$ during the passage through the layer and the mentioned transfer rate.

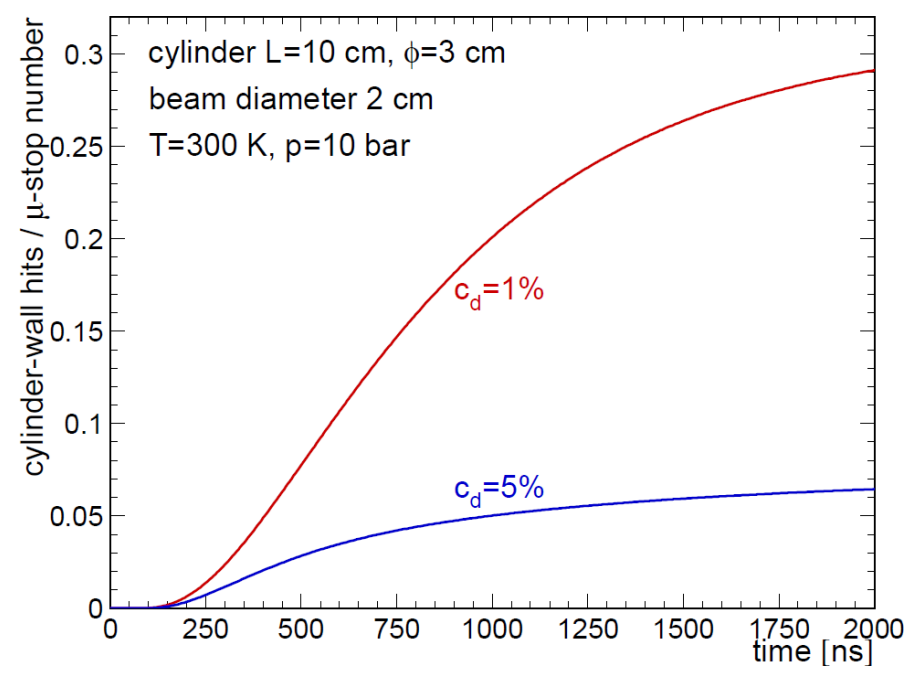

Figure 1. Time-dependent total integrated probability (per stopped muon) that the $\mu \mathrm{D}$ atoms hit the walls of a cylindrical volume with length $\mathrm{L}$ and diameter $\phi$. The red curve and the blue curves show the calculation for a deuterium concentration $\mathrm{c}_{d}$ of $1 \%$ and $5 \%$, respectively.

In order to have a preliminary estimation of the rate of formation of the $\mu \mathrm{Z}$ atoms one has also to take into account the probability of the $\mu \mathrm{D}$ atoms to reach the wall and the solid-angle coverage of the high- $Z$ target. Figure 1 shows the time-dependent total integrated probability that the formed $\mu \mathrm{D}$ atoms reach the walls of a cylindrical volume of $3 \mathrm{~cm}$ diameter and $10 \mathrm{~cm}$ length. The simulation assumes an initial muon beam of $2 \mathrm{~cm}$ diameter stopped uniformly within that volume and it includes the transfer probability $\mu \mathrm{H} \rightarrow \mu \mathrm{D}$, the collisional cross-section $\mu \mathrm{D}+\mathrm{H} 2$ and the lifetime of the muons. The results of two different deuterium concentrations in the H2/D2 mixture are shown. A lower deuterium concentration allows the muonic deuterium to travel easily over large distances, thus explaining the larger probability to reach the wall. $\mathrm{Up}$ to $30 \%$ of the formed $\mu \mathrm{D}$ atoms hit the wall of the cylindrical volume in a mixture containing $1 \%$ deuterium.

Taking into account these factors, the probability $P_{\mu Z}$ of formation of the $\mu \mathrm{Z}$ atom per stopped muon 
can be approximated as follows:

$$
P_{\mu Z}=P_{\mu D-h i t s} \times P_{\text {transfer }} \times \Omega_{\text {target }}
$$

where $P_{\mu D \text {-hits }}$ is the probability that muonic deuterium hits the walls, $P_{\text {transfer }}$ is the transfer probability of the muon from the deuterium to the high- $Z$ element and $\Omega_{\text {target }}$ is the percentage of solid angle covered by the high- $Z$ target.

Since micrograms quantities of the high-Z element corresponds to target's area of the order of $1 \mathrm{~cm}^{2}$, it is clear that the size of the cell needs to be minimized in order to maximize the solid-angle coverage of the target.

\section{Recent progress}

The first transfer experiment with the technique described in the previous section was performed in the $\pi \mathrm{E} 5$ beam line of the Paul Scherrer Institut. A negative muon beam of $20 \mathrm{MeV} / \mathrm{c}$ selected momentum and typical intensity of $10^{4} \mathrm{~s}^{-1}$ was used. The experimental setup consisted of a cylindrical aluminum cell, $3 \mathrm{~cm}$ diameter and $10 \mathrm{~cm}$ long, mounted at the end of the $\pi \mathrm{E} 5$ beam line through a kapton window $125 \mu \mathrm{m}$ thick. The cell was filled with 10 bar H2(99\%)/D2(1\%) mixture at room temperature. The cylindrical walls of the cell were covered with a copper foil coated with $50 \mathrm{~nm}$ of ${ }^{197} \mathrm{Au}$. A schematic view of the cell is shown in Fig. 2 (right).
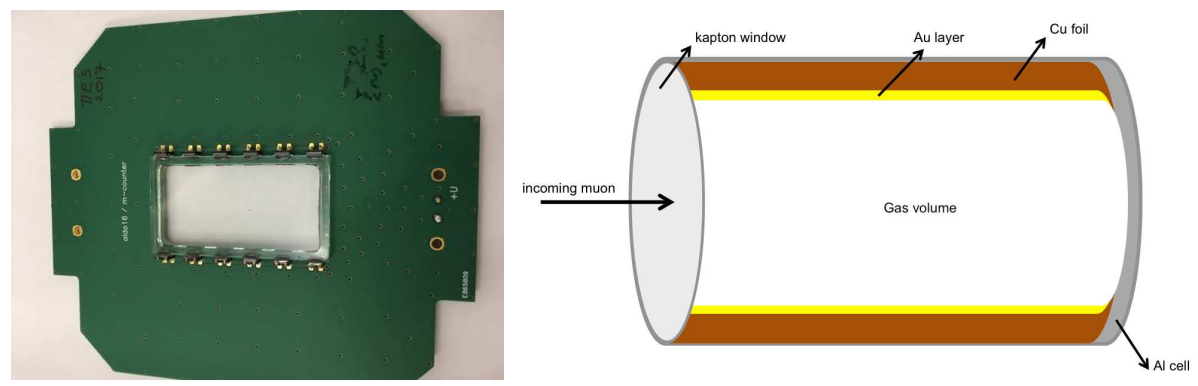

Figure 2. Left: Picture of the BC-400 plastic scintillator used to trigger the entrance of the muons in the cell volume. The twelve silicon photomultipliers used for the readout are visible. Right: Schematic view of the gas cell.

A plastic scintillator (BC-400), $20 \times 40 \mathrm{~mm}^{2}$ and $200 \mu \mathrm{m}$ thick, was mounted inside the beam line $4.5 \mathrm{~cm}$ upstream the entrance of the cell and it was used to trigger the entrance of the muons in the cell volume and for time measurements. The detector was read out by a total of twelve silicon photomultipliers (SiPM) glued along the two long sides and connected in series. A picture of the scintillator detector is shown in Fig. 2 (left).

A HPGe detector with $20 \%$ relative efficiency was used to detect the muonic X-rays and placed in close vicinity to the cell. Figure 3 shows the cell and detector arrangement. The second HPGe detector shown in Fig. 3 did not work during the test.

The occurrence of the double transfer process $\mu H \rightarrow \mu D \rightarrow \mu Z$ can be clearly identified by looking at the time distribution of the muonic X-rays of gold with respect to the time the muon entered the cell given by the entrance scintillator. The muonic X-rays cascade is expected to be delayed over several hundreds of ns due to the time for the transfer process to occur inside the cell.

Figure 4 shows, on a two-dimensional plot energy versus time, the muonic 2 p-1s X-rays in ${ }^{197} \mathrm{Au}$ (left 


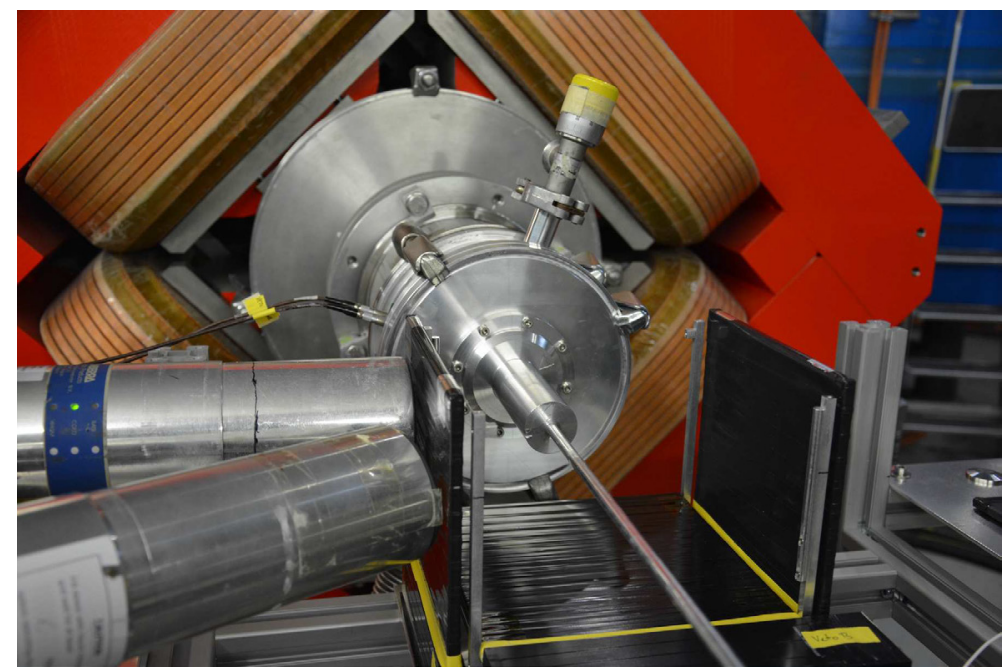

Figure 3. Room temperature hydrogen cell mounted at the end of the $\pi \mathrm{E} 5$ beam line. The last quadrupole magnet of the beam line is visible. The cell was operated routinely at 10 bar hydrogen/deuterium mixtures. A plastic scintillator (in vacuum) was mounted in the beam line in front of the gas cell.

panel) and in ${ }^{63} \mathrm{Cu}$ (right panel). The time is measured relatively to the entrance scintillator. In Fig. 4 left panel, the appearance of the delayed $5764.9 \mathrm{keV} 2 p_{3 / 2} \rightarrow 1 s$ and $5595.0 \mathrm{keV} 2 p_{1 / 2} \rightarrow 1 s \mathrm{X}$-rays in muonic gold demonstrates that the full transfer chain described above is indeed happening in the hydrogen cell with a $1 \%$ deuterium admixture. In the same plot the single and double escape peaks of the $2 \mathrm{p}-1 \mathrm{~s}$ X-rays of muonic gold are also clearly visible at lower energies. These peaks are very pronounced due to the small size of the HPGe detector used. The observed prompt muonic $2 \mathrm{p}-1 \mathrm{~s}$ $\mathrm{X}$-ray transitions in ${ }^{63} \mathrm{Cu}$, at $1508.05 \mathrm{keV}$ and $1514.45 \mathrm{keV}$, shown in Fig. 4 right panel, are from the direct capture of muons which do not stop in the gas and hit directly the walls of the cell stopping in the thick copper layer. These muonic X-rays are emitted within less than hundred nanoseconds after the muon entered the cell.

The time distribution of the muonic ${ }^{197} \mathrm{Au} 2 p_{3 / 2} \rightarrow 1 s$ and $2 p_{1 / 2} \rightarrow 1 s$ X-rays is shown in Figure 5 .

The data analysis is still going on in order to extract more quantitative information like the fraction of muons stopping in the cell, the transfer efficiency to the high- $Z$ element and the rate of formation of muonic gold atoms which will be necessary for the optimization of the system.

\section{Future plans}

As the results of the first transfer test are very encouraging, we will continue the technical development in that direction. In order to optimize the solid-angle coverage of the microgram target located inside the gas cell the size of the cell needs to be minimized. At hydrogen densities corresponding to $10 \%$ of liquid density, stopping distribution of negative muons of $28 \mathrm{MeV} / \mathrm{c}$ momentum with RMS of less than $5 \mathrm{~mm}$ can be reached. The required density of the hydrogen gas can be achieved at 100 bar and room temperature or 10 bar and $30 \mathrm{~K}$. A cell at room temperature with still a sufficiently thin window is certainly an advantage with respect to the cryogenic one as it would greatly simplify the handling and operation of the entire system. 

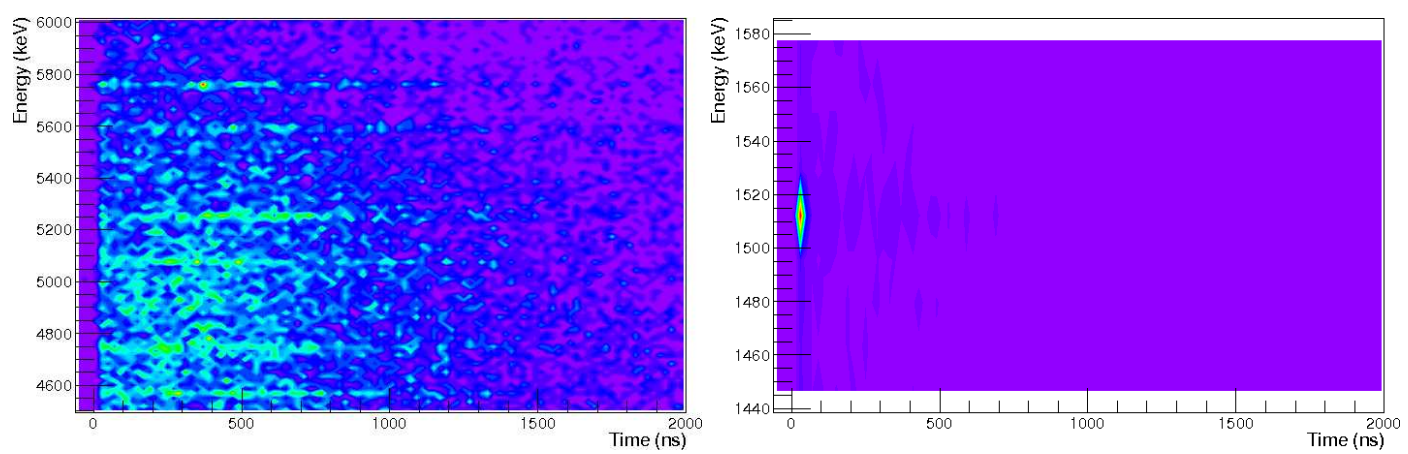

Figure 4. Observed X-ray transitions as a function of time after the muon entered the cell. Left: ${ }^{197} \mathrm{Au} 2 \mathrm{p}-1 \mathrm{~s}$ muonic X-rays at $5764.9 \mathrm{keV}$ and $5595.0 \mathrm{keV}$. The single and double escape peaks are also visible at lower energies. Right: ${ }^{63} \mathrm{Cu} 2 \mathrm{p}-1 \mathrm{~s}$ muonic X-rays at $1508.05 \mathrm{keV}$ and $1514.45 \mathrm{keV}$.



Figure 5. Time distribution of the $2 \mathrm{p}-1 \mathrm{~s} \mathrm{X}$-ray transitions at $5764.9 \mathrm{keV}$ and $5595.0 \mathrm{keV}$ in gold. The time is measured relative to the time of the entrance scintillator.

During 2017, an experimental campaign is planned to test and optimize the transfer reactions method in a cell of $3 \mathrm{~cm}$ diameter and $1.5 \mathrm{~cm}$ length operated at 100 bar and room temperature. The feasibility of the measurement of the muonic X-rays of ${ }^{226} \mathrm{Ra}$ will be demonstrated using $5 \mu \mathrm{g}$ of a stable high-Z target placed on the inner wall of the gas cell. In this configuration, a formation rate of the $\mu \mathrm{Z}$ atoms of approximately $13 \%$ of the incoming muon beam rate is expected.

A large HPGe detector array will be installed in the $\pi \mathrm{E} 1$ experimental area of PSI consisting of $6 \mathrm{HPGe}$ coaxial Ge detectors (60\% relative efficiency) from the UK/French Loan Pool, a Miniball cluster detector [21] and a single crystal HPGe detector (75\% relative efficiency) from KULeuven. Figure 6 
shows a technical drawing of the detector arrangement. The detection efficiency of this array will be around $5 \%$ at $1332 \mathrm{keV}$ and it will greatly enhance the sensitivity to the transfer events.

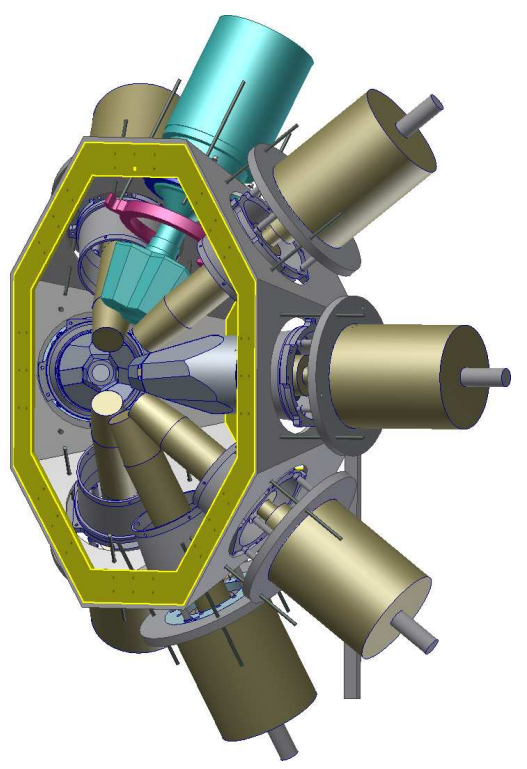

Figure 6. Sketch of the HPGe detectors array which will be used in the 2017 campaign planned at PSI.

\section{Conclusions}

Muonic atoms spectroscopy is a very precise and sensitive tool that allows the determination of the nuclear charge distribution and the deformation properties of nuclei from measured transition energies. It has been an indispensable tool for more than 30 years complementing the measurements of nuclear charge distributions obtained with elastic electron scattering and of differences in mean square radii via isotope shifts using optical laser spectroscopy. The standard way of formation of muonic atoms requires several grams of target material. The possibility to extend muonic atom spectroscopy to elements available in micrograms quantities is pursued at the Paul Scherrer Institut. The method under development is based on the transfer mechanism and the Ramsauer-Townsend effect. In order to cope with the small formation rate of the $\mu \mathrm{Z}$ atoms, a high efficiency, high coverage HPGe array will be used to detect the muonic X-rays. As first physics case, the muonic atom spectroscopy of ${ }^{226} \mathrm{Ra}$ will be performed with the aim to extract the absolute nuclear charge radius of $\mathrm{Ra}$ with a relative precision of $0.2 \%$. This measurement is needed by the experiment currently in progress at the VSI, which aims to measure atomic parity violation effects in single trapped $\mathrm{Ra}^{+}$ions. The method paves the way to the investigation by muonic atom spectroscopy of the nuclear properties of radioactive nuclei with relatively long lifetimes which are usually available or can be safely handled only in small quantities.

This work was supported by the Paul Scherrer Institut through the Career Return Programme, by the Swiss National Science Foundation through the Marie Heim-Vögtlin grant No. 164515 and the project grant No. 200021_165569. The experiment was performed at the $\pi \mathrm{E} 5$ beam line of PSI. We would like to thank the 
accelerator and support groups for the excellent conditions. Technical support by F. Barchetti, F. Burri, M. Meier and A. Stoykov from PSI and B. Zehr from the IPP workshop at ETHZ is gratefully acknowledged. We finally thank A. Weber from PSI for making the Au coating on the $\mathrm{Cu}$ foil.

\section{References}

[1] R. Engfer et al., At. Data Nucl. Data Tables 14, 509 (1974)

[2] G. Fricke and K. Heilig, Nuclear Charge Radii, (Springer-Verlag, Berlin, Heidelberg, 2004)

[3] P. Bergem et al., Phys. Rev. C 37, 2821-2833 (1988)

[4] D.F. Measday, Phys. Rep. 354, 243-409 (2001)

[5] G. Backenstoss et al., Phys. Lett. B 36, 422-424 (1971)

[6] P. Strasser et al. Hyperfine Interact. 119, 317 (1999)

[7] P. Strasser et al. Hyperfine Interact. 193, 121 (2009)

[8] K.P. Jungmann, Hyperfine Interact. 138, 463 (2001)

[9] Wansbeek et al., Phys. Rev. A 78, 050501 (2008)

[10] M.N.Portela et al., Appl. Phys. B 114, 173 (2014)

[11] O.O. Versolato et al., Phys. Rev. A 82, 010501 (2010)

[12] O.O. Versolato et al., Phys. Lett. A 375, 3130 (2011)

[13] G.S. Giri et al., Phys. Rev. A 84, 020503 (2011)

[14] H.-J. Kluge and W. Nörterhäuser, Spectrochim. Acta Part B 58, 1031 (2003)

[15] A. Adamczak et al., At. Data Nucl. Data Tables 62, 255 (1996)

[16] F. Mulhauser et al., Phys. Rev. A 73, 034501 (2006)

[17] A. Adamczak and J. Gronowski, Eur. Phys. J. D 41, 493 (2006)

[18] A. Adamczak, Phys. Rev. A 74, 042718 (2006)

[19] G. Fiorentini and G. Torelli, Nuovo Cimento 36, 317 (1976)

[20] L. Bracci and G. Fiorentini, Nuovo Cimento 50, 373 (1979)

[21] N. Warr et al., Eur. Phys. J. A 49, 40 (2013) 